\title{
The effect of solvent dynamics on the low frequency collective motions of DNA in solution and unoriented films
}

\author{
K.N. Woods, ${ }^{1 *}$ S.A. Lee, ${ }^{2}$ H.-Y. N. Holman, ${ }^{1} \&$ H. Wiedemann ${ }^{3}$ \\ ${ }^{1}$ Ecology Department, Lawrence Berkeley National Laboratory, Berkeley, CA 94720 \\ ${ }^{2}$ Department of Physics \& Astronomy, University of Toledo, Toledo, OH 43606 \\ ${ }^{3}$ Applied Physics Department and SSRL, Stanford University, Stanford, CA 94309
}

Infrared spectroscopy is used to probe the dynamics of in vitro samples of DNA prepared as solutions and as solid unoriented films. The lowest frequency DNA mode identified in the far-infrared spectra of the DNA samples is found to shift in frequency when the solvent influence in the hydration shell is altered. The lowest frequency mode also has characteristics that are similar to $\beta$ - relaxations identified in other glass forming polymers.

\section{Introduction}

Replication and transcription both involve the separation of the two strands of the DNA molecule. Though the mechanism of this strand separation has not yet been elucidated, it has been suggested that the low frequency $\left(<100 \mathrm{~cm}^{-1}\right)$ modes play an important role in this process. ${ }^{1}$ Raman $^{2-7}$ and infrared (IR) studies $^{8}$ have been published for this low frequency region. These experiments have revealed vibrational modes at about $25,40,75,95$, and $115 \mathrm{~cm}^{-1} \cdot{ }^{5,7}$ Experimental evidence has shown that the three

\footnotetext{
*Electronic mail: knwoods2@lbl.gov
} 
highest frequency modes are intramolecular. ${ }^{7}$ Theoretical calculations suggest that these modes involve interbase hydrogen bond breathing motions. ${ }^{9,10}$

The modes in the $\leq 50 \mathrm{~cm}^{-1}$ spectral region, the main subject of this study, are believed to be mainly intramolecular but with detectable intermolecular components. ${ }^{7}$ These modes will be referred to as DNA intermolecular modes. Earlier experimental investigations have described the lowest frequency mode at around $20 \mathrm{~cm}^{-1}$ in film samples of DNA as a libration of stacked bases whose frequency is affected by both intra- and intermolecular interactions. ${ }^{4,5}$ In low frequency Raman experiments, the lowest frequency DNA mode has been found to undergo a slight shift in frequency with a change in counterion concentration or with the degree of hydration. ${ }^{7}$ The shift in frequency of this mode has been attributed to the extent of intermolecular interactions occurring between DNA molecules in the experimental sample. ${ }^{11}$ From these earlier experimental studies, it was concluded that the lowest frequency DNA mode is coupled with the relaxation dynamics taking place within the solvent. ${ }^{5}$ These earlier experiments were performed on solid films or gels of DNA. Such highly concentrated samples of DNA were necessary since the Raman and IR signals are very weak from DNA. However, it is important to perform experiments on DNA in low concentration solutions in order to observe signals associated with the individual DNA molecules themselves and not signals a priori due to aggregations of DNA molecules. Therefore in an effort to develop a deeper understanding about the nature of the DNA intramolecular modes, we have prepared samples of DNA as dilute solutions $(1 \mathrm{mg} / \mathrm{mL})$ and unoriented films to be studied by infrared spectroscopy. 


\section{Experiment}

\section{A. Sample Preparation}

Calf-Thymus Na-DNA, purchased from Sigma-Aldrich, was initially ethanol precipitated to remove excess salt. The unoriented film samples were prepared by resuspending the calf-thymus $\mathrm{Na}-\mathrm{DNA}$ pellet in a solution of $10 \mathrm{mM} \mathrm{NaH}_{2} \mathrm{PO}_{4}$ and $0.01 \mathrm{mM}$ EDTA at a $\mathrm{pH}$ of 7.0. The DNA solution at a concentration of $1 \mathrm{mg} / \mathrm{mL}$ was spread onto a window of silicon in the far-infrared experiments or gold in the midinfrared experiments and placed in an environment with a relative humidity $(\mathrm{RH})$ of $75 \%$. This was accomplished by placing the sample and substrate into a room temperature, sealed glass vessel that had a beaker of saturated $\mathrm{NaCl}$ solution in the bottom. The samples remained in the glass vessel for 1 week before being used in experiment. During the experiment, the DNA film samples were placed into a sealed sample cell that also contained a saturated solution of $\mathrm{NaCl}$ in the bottom in order to maintain $75 \% \mathrm{RH}$ within the sample cell during the collection of the experimental data.

The solution samples were prepared by resuspending the calf-thymus Na-DNA in a solution of $10 \mathrm{mM} \mathrm{NaH}_{2} \mathrm{PO}_{4}$ and $0.01 \mathrm{mM}$ EDTA at $\mathrm{pH} 7.0$. The final concentration of the solution samples was also $\sim 1 \mathrm{mg} / \mathrm{mL}$.

\section{B. Far-infrared measurements}

Coherent FIR radiation produced at the SUNSHINE facility ${ }^{12}$ is used to measure the absorption of film and solution samples of calf-thymus Na-DNA. An electron beam pulse composed of a string of about 3000 equidistant 120 fs-rms bunches every 350 ps is produced at the SUNSHINE facility. ${ }^{13}$ As this beam passes through a thin Al foil, FIR 
Transition Radiation in the form of a Fourier transform limited radiation pulse of equal time structure is produced and is used to measure the absorption and dispersion of film and solution samples of calf-thymus Na-DNA. The spectral range is determined by the Fourier transform of the particle distribution and extends from $5 \mathrm{~cm}^{-1}-120 \mathrm{~cm}^{-1}$. Each micropulse contains energy of $\sim 100 \mathrm{pJ}$. The radiation is coherent and polarized. The frequency-dependent absorption coefficient of the samples is determined from a single, dispersive, interferometric measurement.

The sample cell is composed of a polished, high resistivity silicon window that also serves as a reflector in one arm of the two-beam interferometer. In the experiment, the sample is placed on the backside of the cell window. When radiation enters into the interferometer it is split into two parts by a $0.0254 \mathrm{~mm}$ thick Mylar beam splitter. One beam is reflected onto a movable mirror and the other is transmitted through the beam splitter onto the sample cell. The part of the beam that is transmitted onto the sample cell consists of a ray reflected from the front surface of the silicon window and a delayed pulse reflected from the silicon-sample interface. At normal incidence, the FourierTransform of the ratio of these two signals can be related to the complex Fresnel coefficients. ${ }^{14}$ If the thickness and dielectric properties of the cell window are known, measurement of the frequency-dependent change in the amplitude and phase of the reflected signals allow the optical properties of the sample to be determined. ${ }^{15}$ In these experiments, a room temperature pyroelectric detector (Molectron $\mathrm{LiTaO}_{3} \mathrm{P} 1-65$ detector) is used to detect the coherent FIR signal. In each scan an average of 30 pulses is used for each individual data point in the interferogram and five independent scans are averaged together for each absorption measurement. 


\section{Mid-infrared measurements}

The DNA samples were measured by synchrotron Fourier Transform Infrared (FTIR) spectro-microscopy at the Advanced Light Source at Lawrence Berkeley National Laboratory at beamline $1.4 .^{16}$ The film spectra were collected in the $4000-650 \mathrm{~cm}^{-1}$ spectral region with a diffraction-limited IR beam with a diameter ranging from $3-10$ $\mu \mathrm{m}$. The spectra were collected in reflection mode using a sealed sample cell with a ZnSe window and a MCT-B detector. Gold coated glass slides were used for the sample substrate. Each point in the DNA spectral maps is composed of 128 averaged scans with a data point spacing of $0.482 \mathrm{~cm}^{-1}$.

\section{Normal mode Analysis}

A normal mode analysis (NMA) on fragments of A- form and B- form DNA were performed using the molecular modeling toolkit MMTK (http://starship.python.net/crew/hinsen/MMTK/). The A- form fragment of DNA consists of 20 nucleotides (10 base pairs) and is hydrated with 298 water molecules and 18 sodium ions. The B- form system is composed of 24 nucleotides (12 base pairs), 459 water molecules and 22 sodium atoms. The solvent around both forms of DNA is equivalent to a $5 \AA$ hydration shell surrounding the DNA.

The normal modes provide an analytic description of the vibrational frequencies in the system by using a harmonic approximation near a minimum of the potential. To reach a local minima in the systems studied a conjugate gradient minimization algorithm was utilized. Both DNA systems were energy minimized using the AMBER 94 force field ${ }^{17}$ up to a gradient RMS of $10^{-4} \mathrm{KJ} / \mathrm{mol} / \mathrm{nm}$. In the analysis the normal modes were restricted to the low frequency modes. These are modes associated with the large, 
concerted structural rearrangements taking place in the DNA system. This was accomplished by creating a basis vector from the full Cartesian space of the system that eliminated the fast degrees of freedom such as bond fluctuations.

\section{Normal Modes}

In addition to the experimental analysis, a normal mode study aimed at understanding the origin of the largest amplitude modes of hydrated fragments of DNA has been carried out in our investigation of DNA dynamics. The modes of both A- and B- forms of DNA in the $0-100 \mathrm{~cm}^{-1}$ region are listed in table 1 . We have utilized both forms of DNA in the analysis mainly as a means of contrasting the bands in our experimental film samples, which are a mixture of both forms of DNA, with the normal modes that would be present in either or both forms of DNA. It is also worth noting that in the experimental solution samples, the DNA is exclusively in the B - form.

In both forms of DNA the lowest frequency normal mode from the analysis, 8.82 $\mathrm{cm}^{-1}$ in the B-form and $13.71 \mathrm{~cm}^{-1}$ in the $\mathrm{A}$ - form, can be ascribed to interactions between solvent molecules in the DNA hydration shell. We have found that the higher frequency modes in the $\leq 50 \mathrm{~cm}^{-1}$ region can be attributed to DNA motions that are influenced by the solvent environment (i.e. they have an intermolecular component). The lowest frequency DNA mode $\left(20.86 \mathrm{~cm}^{-1}\right.$ and $25.71 \mathrm{~cm}^{-1}$ in the B-form and A-form respectively) from this analysis can be described as a librational mode involving the entire DNA molecule. This is consistent with the description provided for the lowest frequency DNA mode in earlier analyses. ${ }^{4,5,18}$ The higher frequency modes within the $\leq$ $50 \mathrm{~cm}^{-1}$ energy range originate from torsional fluctuations within the DNA helix. From the analysis, modes with frequencies in the $50-100 \mathrm{~cm}^{-1}$ range have been identified as 
DNA intramolecular modes. The DNA intramolecular modes are characterized by motions involving hydrogen bonding interactions between the base-pairs in the DNA duplex and the phosphate groups on the DNA backbone; and are consistent with modes identified in previous experimental and simulation studies on collective motions in DNA. $^{7,10,18}$

It should also be noted that peaks from the pure solvent (mostly water in this study) appear in the FIR absorption spectrum. The peaks from the solvent are attributed to bending and torsional motions associated with the local structure of liquid water and are located in the $55 \mathrm{~cm}^{-1}-70 \mathrm{~cm}^{-1}$ spectral region. The presence of these features complicates the analysis of the DNA modes in this region; consequently, we will be concerned mainly with the FIR absorption modes below $50 \mathrm{~cm}^{-1}$ in this study.

\section{Relaxation dynamics and DNA vibrational modes}

\section{A. Relaxation processes in glass forming liquid systems}

Local structural relaxation occurs over a broad range of time scales. In a glass forming liquid relaxation takes place in stages. The initial stage of the relaxation processes begins with a fast inertial decay that is followed by a relaxation at intermediate times. The intermediate time relaxation, sometimes referred to as a $\beta$ - process or $\beta$ relaxation in mode coupling theory (MCT) ${ }^{19}$ occurs on the picosecond time scale (on the order of a few picoseconds) and has been described as a localized intramolecular orientational motion ${ }^{20}$ (not to be confused with the DNA intramolecular modes discussed in section III) that takes place within a minima of the potential energy surface. In dielectric spectroscopy and low frequency Raman scattering experiments the $\beta$ processes can appear as a broad distribution of relaxation rates ${ }^{21}$ in the spectrum; and are 
neither wave vector dependent $t^{22}$ nor strongly temperature dependent. ${ }^{23}$ The final stage of the relaxation processes is characterized by a long time decay in which the system undergoes a transition between distinct minima. ${ }^{24}$ The long time decay or $\alpha$-process occurs on a much longer timescale, usually hundreds of picoseconds to milliseconds depending on environmental conditions, and ends in conformational change. Results from both theoretical and experimental analyses on many glass forming liquids have indicated that $\beta$ - type relaxations reduce the effective energy barrier for longer time structural relaxation. ${ }^{25}$ Consequently, the lowest frequency DNA mode uncovered in early Raman and FIR experiments on DNA film samples possess characteristics similar to those associated with $\beta$ - type processes identified in mode coupling theory. The FIR spectra of DNA in solution and as an unoriented film at $298 \mathrm{~K}$ are shown in Fig. 1. Modes in the $\leq 50 \mathrm{~cm}^{-1}$ region of the film spectrum (labeled $1-3$ ) are identified at approximately $25 \mathrm{~cm}^{-1}, 35 \mathrm{~cm}^{-1}$, and $47 \mathrm{~cm}^{-1}$; while those in the $>50 \mathrm{~cm}^{-1}$ region of the FIR spectrum (labeled 4-6) are found at about $65 \mathrm{~cm}^{-1}, 79 \mathrm{~cm}^{-1}, 87 \mathrm{~cm}^{-1}$, and $95 \mathrm{~cm}^{-1}$. The equivalent modes are labeled in the solution sample FIR spectrum. Interestingly, an analysis of the normal modes specifically related to DNA backbone motion has revealed that a strong backbone mode (involving the phosphate groups) oscillates at different frequencies in the two forms of DNA studied. The backbone mode in the normal mode analysis is found at about $81 \mathrm{~cm}^{-1}$ in the $\mathrm{B}-$ form of DNA and at $86 \mathrm{~cm}^{-1}$ in the $\mathrm{A}-$ form. The similarity of the peaks in the same region in the experimental spectra of the DNA samples (in Fig. 1) has encouraged us to identify the bands labeled as $5 \mathrm{a}$ and $5 \mathrm{~b}$ in the experimental film spectrum (which is a mixture of both forms of DNA) and 5 in the solution sample with DNA backbone motions. 


\section{B. Influence of the solvent dynamics on the biomolecule dynamics}

Experimental measurements on the diffusion properties of solvents in and around various enzymes and proteins have established that conformational changes within the proteins are accompanied by a net change of solvent molecules into and from the hydration shell into the bulk. ${ }^{26}$ Additionally, numerous experimental studies on proteins have demonstrated that viscosity of the solvent has a strong influence on the reaction rates of chemical processes taking place within the protein. ${ }^{27}$ Both examples, although focusing on different aspects of protein dynamics, illustrate that solvent dynamics are somehow closely connected with the dynamics of the biological molecule being studied. An examination of the role of solvent in protein dynamics ${ }^{28}$ has categorized proteinsolvent coupled motions into two categories: hydration shell coupled motions or $\beta$ fluctuations, where the protein motion is coupled to the fast (picosecond) fluctuations of the solvent molecules in the protein hydration shell and solvent slaved motions or $\alpha-$ fluctuations where large scale protein motions are coupled to dielectric changes in the bulk solvent. Ultrafast studies ${ }^{29}$ on the hydration dynamics of water molecules on both the surface and in the grooves of DNA have revealed a similar relationship between solvent and DNA dynamics. The correlation time of water molecules in the DNA hydration shell could be separated into those weakly bound to the DNA (20 ps) and those fluctuating between the DNA hydration shell and the solvent bulk (1.5 ps). The time scale of the correlation times identified in the DNA hydration dynamics studies are

consistent with those uncovered in earlier experimental studies of DNA dynamics. ${ }^{5,6}$ For years it has been recognized that water concentration or activity in the DNA hydration shell has a strong influence on DNA conformation. Highly hydrated Na-DNA is 
normally found in the B- form while low humidity or DNA under high salt concentrations favor the A-form and Z- form respectively. ${ }^{30}$ The conformational transition between one form of DNA and another is believed to be dynamically driven by a change in the solvent conditions. The solvent not only hydrates the biological molecule but also provides a variable reservoir of entropy in the macromolecule hydration shell. Based on experimental investigations on proteins and nucleic acids, it is likely that the net flux of solvent molecules into and out of the hydration shell may be important for facilitating intermolecular interactions between macromolecules, in addition to providing a hydrogen bonding base for actual macromolecule association. ${ }^{26}$ For instance, a change in solvent activity of the enzyme EcoRI has been shown to affect the site specific recognition of the enzyme with its DNA substrate. ${ }^{31}$ In the particular case of DNA hydration, the time scale of the more mobile water molecules (1.5 ps), identified in the ultrafast hydration dynamics studies, is slightly longer than the time scale of hydrogen bond breakage in the solvent. Particularly under these circumstances, it is possible to envision how fast fluctuations within the solvent might also modify the effective potential energy surface of the biological molecule in which it interacts. Reconsidering the FIR spectra in Fig. 1, there is noticeable shift in the lowest frequency DNA mode from approximately $25 \mathrm{~cm}^{-1}$ in the film sample to about $16 \mathrm{~cm}^{-1}$ in the solution sample indicating that interaction with the solvent molecules in the fully hydrated DNA sample has altered the dynamics of the lowest frequency mode. The higher frequency modes in both spectra are remarkably similar. These results are consistent with earlier Raman studies that have attributed the shift in the lowest frequency DNA mode to the weakening of the intermolecular bonds between DNA molecules due to the greater number of solvent molecules in the hydration 
shell. ${ }^{11}$ On the other hand, these results are also consistent with the hypothesis that solvent coupled motions lower the energy barrier for conformational motions taking place within the macromolecule. ${ }^{32}$

\section{Solvent hydrogen bonding and the influence on DNA dynamics}

More recent experimental results focusing on the more detailed aspects of protein dynamics have demonstrated that solvent viscosity may not be the only important factor determining overall protein dynamics. ${ }^{33}$ Recent light scattering studies on the conformational fluctuations in both lysozyme and myoglobin have determined that fast fluctuations occurring in the solvent (on the picosecond time scale) are directly connected with the rate of biochemical reactions taking place within the two proteins. ${ }^{34}$ Based on these experimental observations it has become more apparent that solvent properties do not simply define the long time reorientation characteristics of the protein; but solvent activity also appears to have an important affect on the short-time dynamics determining protein activity on the picosecond time scale. In many complex macromolecular associations a conformational change, which is required to carry out a specific biochemical process, may involve the simultaneous or collective relaxation of several modes. For instance, in an analysis of CO- myoglobin association, it has been suggested that fluctuations within the solvent network may be important for facilitating the sequential conformational fluctuations in the segments that are ultimately necessary for large conformational changes in the protein. ${ }^{28}$ As the temperature decreases, the time scales of these motions increase along with the structural or $\alpha$-relaxation time of the solvent. Earlier Raman studies on hydrated film samples of Na-DNA observed that lowering the film sample temperature results in a blue-shift in frequency of the lowest 
frequency DNA mode. ${ }^{3}$ In these studies the shift in frequency in the Raman spectrum was attributed to the increased strength of intermolecular interactions between adjacent DNA molecules at the lower temperature, which in turn had an effect on the frequency of the lowest frequency DNA mode. In Fig. 2, the lowest frequency mode in the 75\% RH unoriented film samples at $298 \mathrm{~K}$ and $270 \mathrm{~K}$ from this study show a remarkably different behavior when compared to earlier Raman studies. The lowest frequency mode at $298 \mathrm{~K}$ shifts from approximately $30 \mathrm{~cm}^{-1}$ in the FIR spectrum to $18 \mathrm{~cm}^{-1}$ at $270 \mathrm{~K}$, suggesting that there may be other mechanisms affecting the dynamics of this mode in the FIR unoriented film spectra. This is a topic that will be explored further in the following paragraphs.

From dielectric relaxation spectroscopy measurements on solution samples of $\mathrm{Na}$ DNA, it has been determined that as the temperature of the sample is lowered ion mobility of the solvent decreases. ${ }^{35}$ Additionally, reduction in temperature was also found to lead to a decrease in DNA chain length as a result of a change in the solvent activity around the DNA. Interestingly, a similar trend relating to DNA chain length was observed in the same study when the DNA concentration was increased; implying that both temperature as well as concentration changes in the DNA sample are capable of altering the solvent activity within the DNA hydration shell. Molecular dynamics studies on other glass forming polymers have also identified a decrease in polymer chain length when interactions, in the form of hydrogen - bonding, with the solvent are reduced. ${ }^{36}$ Consequently, the decrease in polymer-solvent interaction also results in enhanced translation diffusion of the polymer chains, in addition to increased orientational mobility within the polymer chain segments. The peaks in the $1430-1415 \mathrm{~cm}^{-1}$ region of the mid- 
infrared (MIR) spectra of a DNA film sample at temperatures ranging from $278 \mathrm{~K}$ to 298 K (Fig. 3) indicate that the solvent molecules in the hydration shell are distributed differently about the DNA at colder temperatures when compared to the room temperature sample. The peaks in this region are sensitive to the sugar conformation in the DNA molecules with respect to the base orientation and are influenced by the organization of the solvent around the helix. The peak at approximately $1419 \mathrm{~cm}^{-1}$ is typical of the $\mathrm{C}^{3}$ '-endo/ anti geometry found in A -type molecules while the $1425 \mathrm{~cm}^{-1}$ band reflects the $\mathrm{C} 2$ '-endo/ anti geometry commonly found in $\mathrm{B}-$ form molecules. ${ }^{37,38}$ As was previously mentioned in section III, the DNA film samples in this study are composed of a mixture of A- and B-form type DNA. In Fig. 3 there appears to be a gradual shift in preference of the B- type geometry at colder temperatures to the A-type geometry at room temperature suggesting a change in the distribution of solvent molecules in the primary hydration shell. Additionally, a shoulder in the $278 \mathrm{~K}$ spectrum around $1422 \mathrm{~cm}^{-1}$ suggests that an intermediate conformation exists at that temperature that is not present at the other temperatures. Molecular dynamics studies focusing on the underlying mechanisms contributing to DNA aggregation ${ }^{39}$ have determined that at colder temperatures long-lived water molecules in the grooves of the DNA molecules facilitate intermolecular interactions by screening the repulsive interactions between phosphate groups along the DNA backbone. Furthermore, the long-lived water molecules were also found to serve as a bridge in stabilizing the DNA intermolecular interactions by forming hydrogen bonds between adjacent bases. In in vitro experiments DNA aggregation has also been facilitated by the use of salts which alter the electrostatic environment around the DNA molecules. ${ }^{40}$ In Fig. 4 peaks in the $1725 \mathrm{~cm}^{-1}-1705$ 
region of MIR spectra of a DNA film sample at varying temperatures indicates that an increased ability to form DNA intermolecular hydrogen bonds exists at lower temperatures. The peak at $1718 \mathrm{~cm}^{-1}$ in the MIR spectra reflects the $\mathrm{C}-\mathrm{O}$ stretching vibration of G-C base pairs in the DNA molecules. The additional peak at $1713 \mathrm{~cm}^{-1}$, most apparent at $278 \mathrm{~K}$, indicates the presence of intermolecular base pairing between molecules in the film sample. As an aside, the appearance of the $1713 \mathrm{~cm}^{-1}$ band in the MIR spectrum of the DNA film is accompanied by a decrease in a band at $1623 \mathrm{~cm}^{-1}$ (not shown) due to adenine ring vibrations, which is another indication of DNA intermolecular associations. $^{38}$

Returning to the discussion regarding the FIR spectra of the film samples in Fig. 2 , it is possible that the red-shift of the lowest frequency mode can be explained in terms of solvent influence on the DNA dynamics. With stronger hydrogen bonds between the DNA and the solvent, the lowest frequency mode of DNA would be expected to be hindered due to a higher energy barrier for reorientational motion. In Fig. 2 there is a noticeable decrease in frequency of the lowest frequency mode in the $270 \mathrm{~K}$ film sample when compared with the room temperature film sample suggesting that the solvent influence, in terms of solvent hydrogen bonding, in the DNA dynamics is decreased at $270 \mathrm{~K}$. In a molecular dynamics study of dielectric relaxation in another chain forming liquid it was determined that decrease in solvent influence on the chain dynamics of the molecule lead to a greater contribution of translational diffusion in the relaxation process. ${ }^{41}$ This was mainly due to the absence of the high frequency librational modes of the solvent being available to help accelerate the decay of the dipole density correlations. The translational contribution was found to arise mainly in the form of low frequency 
librations within the hydrogen bonded chains of the molecule. Its presence was most apparent in the intermediate time scale of the relaxation process, where fast reorientation fluctuations within the chains lead to faster decay of the correlations on the (few) picosecond time scale; although the longer time decay, leading to the main or $\alpha$ relaxation was significantly slower when contrasted with conditions in which the solvent molecules interacted more directly with the solute.

The spatial dependence of the intermolecular interactions taking place in a DNA film sample at different temperatures is shown in the MIR spectro-microscopy image in Fig. 5. In this figure the $1713 \mathrm{~cm}^{-1}$ peak is used as a visual marker to highlight the intermolecular interactions in the DNA film. From the image it becomes clear that there are regions of very highly concentrated DNA associations in the film sample, which would also imply a reduced amount of solvent in those regions. This phenomenon is most apparent in the film sample at the lower temperatures investigated and is still visible but mostly dissipated in the room temperature image. Lower solvent activity results in a change in the conformational entropy in the biomolecule hydration shell. For example, both gene expression and DNA recombination are cellular processes that initially require a restriction of large conformational motions in the intracellular matrix in favor of more ordered association to proceed.

A comparison of the FIR spectra of the solution samples of DNA at $270 \mathrm{~K}$ and $298 \mathrm{~K}$ in Fig. 6 suggest that the additional solvent modes available in the more hydrated samples are also capable of altering the DNA dynamics. In the $\leq 50 \mathrm{~cm}^{-1}$ region of the experimental spectra the lowest frequency DNA mode is the same within experimental uncertainty at both temperatures; but there is a noticeable red-shift of the higher 
frequency modes (in the $\leq 50 \mathrm{~cm}^{-1}$ region) in the $270 \mathrm{~K}$ solution spectrum when compared with the room temperature solution sample. The higher frequency modes in this same spectral region $\left(\leq 50 \mathrm{~cm}^{-1}\right)$ do not vary significantly in the FIR spectra of the other DNA samples already considered in previous sections. The DNA motions in the $\leq$ $50 \mathrm{~cm}^{-1}$ region have previously been identified as intermolecular modes in the normal mode analysis covered in section III. The time scale of the solvent correlations in the DNA hydration shell are dependent on either the proximity or type of association that the solvent has with the biomolecule. Within this context it becomes clear that there are a myriad of relaxation processes in the solvent that can influence biomolecule dynamics. Recent experimental investigations on glass forming liquids with $\beta$ - relaxations have confirmed that molecules with internal degrees of freedom can possess $\beta$ - type relaxations that are spatially heterogeneous. ${ }^{42}$ The heterogeneity in the process appears as a distribution of time scales in the $\beta$-relaxation with slower and faster contributions. In section IVA. it was mentioned that the lowest frequency DNA mode possess characteristics similar to those associated with $\beta$ - type processes found in other glass forming liquids ; and we have identified the other bands in the $\leq 50 \mathrm{~cm}^{-1}$ region as torsional motions with the DNA double helix. In this scenario, it is possible that the torsional motions are also involved in the ( $\beta$-) relaxation process. Modifying the DNAsolvent interactions of the more 'bulk-like' molecules in the extended hydration shell of the solution samples in Fig. 6 may also promote associations between the molecules that require a modification or accentuation of modes related to DNA conformational fluctuations. This is a subject that will require further investigation in the future. 


\section{Discussion}

In our experiments we have facilitated a change in solvent activity, which we have used synonymously with solvent influence throughout this discussion, by varying the temperature and degree of hydration in the DNA samples studied. In an in vivo system it is likely that the amount of solvent present in the cell would be much lower than that used in our in vitro experimental samples. Small changes in volume or concentration, which would also alter the solvent activity, would undoubtedly have a considerable affect on the activity of macromolecules sharing the same hydration shell. The unusual properties of the solvent, which in this study is essentially water and also the most likely solvent in vivo, may also play a significant role in determining the types of motions the macromolecules experience in the hydration shell.

One issue that has not been addressed in the discussion regarding the experimental FIR spectra of the DNA films in this study is why are results differ considerably from previous Raman and FIR studies. The differences in the experimental spectra are most apparent in the frequency of the lowest frequency DNA mode. One explanation can be ascribed to the manner in which the samples have been prepared in our experiments when compared to earlier experiments on DNA films. We have prepared unoriented DNA film samples where the molecules in such films come in close contact only at random points. This contrasts with the many contact points for DNA in ordered samples in which the DNA molecules are aligned in a parallel fashion. It is possible that the oriented film samples possess stronger intermolecular correlations than in the unoriented films which may possibly explain the discrepancy between our results and earlier studies. 


\section{Conclusions}

We have used infrared spectroscopy to explore the dynamics in DNA film and solution samples. From our measurements we have found that the lowest frequency DNA mode is affected by the interactions with the solvent in the hydration shell. Lowering the temperature of the film samples leads to an increased intermolecular attraction between the DNA molecules and a decrease in frequency of the lowest frequency DNA mode. We have explained this shift in frequency in terms of a reduced solvent influence in the DNA hydration shell. We have also speculated that reduced solvent influence may be significant for the initiation of certain cellular processes occurring in in vivo systems and may be a phenomenon unique to the solvent (water) used in these experiments.

From our measurements we also find that at room temperature the solvent influence is higher when compared to the colder temperature samples. Addition of solvent molecules in the DNA hydration shell under these conditions (high solvent influence) leads to faster relaxation of the lowest frequency DNA mode when compared to a sample with less hydration. These results are consistent with other protein and nucleic acid studies that have determined that the solvent reduces the energy barrier for fast picosecond conformational fluctuations occurring in the biomolecule.

Throughout the discussion on the DNA dynamics explored in this study and from the results of previous studies on DNA, we have noted the lowest frequency mode detected has similar characteristics to $\beta$ - type relaxations (Johari-Goldstein type) identified in other glass forming polymers. Within this context, it would be interesting to gain greater insight into the response of this mode under a range of experimental 
conditions as well as its response with other macromolecules. It is possible that a deeper understanding about the dynamics of this mode may further elucidate the conformational changes that occur in the DNA molecules during biochemical processes and other associations with macromolecules in the cell.

\section{ACKNOWLEDGEMENTS}

This work is supported in part by the U.S. Department of Energy under contract number DE-AC02-76SF0051 at SLAC and by the Directors of the Office of Science, Office of Biological and Environmental Research, Medical Science Division, Materials Science Division, of the United States Department of Energy under contract number DE-AC03-76SF00098 at LBNL. We would also like to thank Professor Carlos Guestrin for the generous use of computational time for carrying out the normal mode analyses. 


\section{References}

1 E. W. Prohofsky, Phys. Rev. A 38, 1538 (1988); E. W. Prohofsky, Comments Mol. Cell. Biophys. 2, 65 (1983).

2 H. Urabe and Y. Tominaga, J. Phys. Soc. Jpn. 50, 3543 (1981); H. Urabe and Y. Tominaga, Biopolymers 21, 2477 (1982); S. M. Lindsay, S. A. Lee, J. W. Powell, T. Weidlich, C. DeMarco, G. D. Lewen, N. J. Tao, and A. Rupprecht, Biopolymers 27, 1015 (1988); T. Wiedlech and S. M. Lindsay, Phys. Rev. Lett. 61 (14), 1674 (1988); T. Weidlich and S. M. Lindsay, J. Phys. Chem 92, 6479 (1988).

3 H. Urabe, M. Kato, Y. Tominaga, and K. Kajiwara, J. Chem. Phys. 92, 768 (1990).

$4 \quad$ H. Urabe, Y. Sugawara, M. Tsukakoshi, and T. Kasuya, J. Chem. Phys. 95, 5519 (1991).

5 H. Urabe, Y. Sugawara, M. Ataka, and A. Rupprecht, Biophysical J. 74, 1533 (1998).

6 N. J. Tao, S. M. Lindsay, and A. Rupprecht, Biopolymers 27, 1655 (1988).

7 T. Weidlich, S. M. Lindsay, O. Rui, A. Rupprecht, W. L. Peticolas, and G. A. Thomas, J. Biomolec. Str. Dyn. 8, 139 (1990).

8 A. Wittlin, L. Genzel, F. Kremer, S. Haeseler, A. Poglitsch, and A. Rupprecht, Phys. Rev. A 34, 493 (1986); J. W. Powell, G. S. Edwards, L. Genzel, F. Kremer, A. Wittlin, W. Kubasek, and W. Peticolas, Phys. Rev. A 35, 3929 (1987); T. Weidlich, J. Powell, L. Genzel, and A. Rupprecht, Biopolymers 30, 477 (1990). 

(1984).

Y. Z. Chen and E. W. Prohofsky, Biopolymers 35, 573 (1995).

T. Weidlich, S. M. Lindsay, and A. Rupprecht, Phys. Rev. Lett. 61, 1674 (1988).

P. Kung, H. C. Lihn, D. Bocek, and H. Wiedemann, Phys. Rev. Lett. 73, 967 (1994).

H. C. Lihn, D. Bocek, M. Hernandez, P. Kung, C. Settakorn, and H. Wiedemann, Phys. Rev. E 53, 6413 (1996); H. Wiedemann, D. Bocek, M. Hernandez, and C. Settakorn, J. Nucl. Mat 248, 374 (1997).

M. N. Afsar and J. B. Hasted, Infrared Physics 18, 835 (1978).

J. R. Birch and M. Bennouna, Infrared Physics 21, 229 (1981).

M. C. Martin and W. R. McKinney, Proc. Mater. Res. Soc. 524, 11 (1998); H.-Y.

N. Holman, M. C. Martin, and W. R. McKinney, Spectroscopy - An International Journal 17 (2-3), 139 (2003).

D. A. Pearlman, D. A. Case, J. W. Caldwell, W. R. Ross, I. T. E. Cheatham, S. DeBolt, D. Ferguson, G. Seibel, and P. Kollman, Comp. Phys. Commun. 91, 1 (1995).

S. Cocco, J. Chem. Phys. 112 (22), 10017 (2000).

W. Gotze, J. Phys.:Condens. Matter 11, A1 (1999); W. Gotze and L. Sjogren, Rep. Prog. Phys. 55, 241 (1992).

L. Wu, Phys. Rev. B 43, 9906 (1991); A. Arbe, D. Richter, J. Colmenero, and B. Farago, Phys. Rev. E 54, 3853 (1996). 
G. P. Johari and M. Goldstein, J. Chem. Phys. 53, 2372 (1970); G. P. Johari and M. Goldstein, J. Chem. Phys. 55, 4245 (1971). C. A. Angell, J. Non-Cryst. Solids 73, 1 (1985); C. A. Angell, in Relaxation in Complex Systems, edited by K. Nagai and G. B. Wright (National Technical Information Service, U.S. Department of Commerce, Springfield, 1985). M. Kruger, M. Soltwisch, I. Petscherizin, and D. Quitmann, J. Chem. Phys. 96 (10), 7352 (1992).

A. Sjolander, in Static and Dynamic Properties in Liquids, edited by M.

Davidovic and A. K. Soper (Springer, Berlin, 1989), pp. 90.

G. Meier, F. Fujara, and W. Petrys, Macromolecules 22, 4421 (1989).

M. Billeter, P. Guntert, P. Luginbuhl, and K. Wuthrich, Cell 85, 1057 (1996).

A. Ansari, C. M. Jones, E. R. Henry, J. Hofrichter, and W. A. Eatron, Science 256, 1796 (1992); D. Beece, L. Eisenstein, H. Frauenfelder, D. Good, M. C. Marden, L. Reinisch, A. H. Reynolds, and K. T. Yue, Biochemistry 19, 5147 (1980); S. J. Hagen, J. Hofrichter, and W. A. Eaton, J. Phys. Chem 100, 12008 (1996). (40), $14408(2004)$. Zhao, T. Xia, and A. H. Zewail, PNAS 100 (24), 13746 (2003). W. Saenger, W. N. Hunter, and O. Kennard, Nature 324 (6095), 385 (1986).

C. R. Robinson and S. G. Sligar, J. Mol. Biol. 234, 302 (1993). 
P. W. Fenimore, H. Frauenfelder, B. H. McMahon, and F. G. Parak, PNAS 99 (25), 16047 (2002).

T. Kleinert, W. Doster, H. Leyser, W. Petry, V. Schwarz, and M. Settles, Biochemistry 37, 717 (1998); C. Andreani, A. Filabozzi, F. MEnzinger, A. Desideri, A. Deriu, and D. D. Cola, Biophys. J. 68, 2519 (1995). G. Caliskan, D. Mehtani, J. H. Roh, A. Kisliuk, A. P. Sokolov, S. Azzam, M. T. Cicerone, S. Lin-Gibson, and I. Peral, J. Chem. Phys. 121 (4), 1978 (2004). M. Sun, S. Pejanovic, and J. Mijovic, Macromolecules 38, 9854 (2005).

I. Bahar, B. Badur, and P. Donuker, J. Chem. Phys. 99 (3), 2235 (1993); Y. E. Ryabov, Y. Feldman, N. Shinyashiki, and S. Yagihara, J. Chem. Phys. 116 (19), 8610 (2002).

M. Banyay, M. Sarkar, and A. Graslund, Biophysical Journal 104, 477 (2003).

J. Liquier and E. Taillandier, in Infrared Spectroscopy of biomolecules, edited by H. H. Mantsch and D. Chapman (Wiley-Liss, Inc, New York, 1996), pp. 131. L. Yang, I. Lee, C. Wang, Q. Li, and C. Bai, J. Molec. Struct. 442, 275 (1998). S. Flock, R. Labarbe, and C. Houssier, J. Biomol. Struct. Dyn. 13, 87 (1995); J. L. Sikorav, J. Pelta, and F. Livolant, Biophys. J. 67, 1387 (1994); P. G. Arscott, C. Ma, J. R. Wenner, and V. A. Bloomfield, Biopolymers 36, 345 (1995). B. M. Ladanyi and M. Skaf, J. Phys. Chem 100, 1368 (1996). 12, A383 (2000); R. Richert, Europhys. Lett. 54, 767 (2001). 


\section{Table captions:}

\section{Table 1:}

Normal modes (in $\mathrm{cm}^{-1}$ ) of A-form and B-form DNA. The type refers to the origin of the DNA mode: solvent (S), intermolecular (I), or intramolecular (N)

\section{Figure captions:}

Figure 1:

FIR absorption spectra of a solution sample of DNA (filled circles) and an unoriented film sample of DNA at 75\% RH (filled triangles) at $298 \mathrm{~K}$.

\section{Figure 2:}

FIR absorption spectra of a $75 \% \mathrm{RH}$ film sample of DNA at $270 \mathrm{~K}$ (open triangles) and a 75\% RH film sample of DNA at $298 \mathrm{~K}$ (filled triangles).

\section{Figure 3:}

$1430-1415 \mathrm{~cm}^{-1}$ region of the MIR absorption spectra of a $75 \% \mathrm{RH}$ film sample of DNA at $278 \mathrm{~K}$ (solid line), $283 \mathrm{~K}$ (dashed line), $288 \mathrm{~K}$ (dotted line), and $298 \mathrm{~K}$ (dasheddotted line).

\section{Figure 4:}

$1725-1705 \mathrm{~cm}^{-1}$ region of the MIR absorption spectra of a $75 \% \mathrm{RH}$ film sample of DNA at $278 \mathrm{~K}$ (solid line), $283 \mathrm{~K}$ (dashed line), $288 \mathrm{~K}$ (dotted line), and $298 \mathrm{~K}$ (dasheddotted line). 
Figure 5:

MIR spatial area map highlighting the $1713 \mathrm{~cm}^{-1}$ intermolecular DNA band in a $75 \% \mathrm{RH}$ film sample of DNA at
a) $278 \mathrm{~K}$
b) $283 \mathrm{~K}$
c) $288 \mathrm{~K}$
d) $298 \mathrm{~K}$

\section{Figure 6:}

FIR absorption spectra of solution samples of DNA at $270 \mathrm{~K}$ (open circles) and $298 \mathrm{~K}$ (closed circles) 
Table 1

\begin{tabular}{||l|l|l||}
\hline A & B & Type \\
\hline 13.71 & 8.82 & S \\
\hline 25.74 & 20.86 & I \\
\hline 37.76 & 32.89 & I \\
\hline 49.79 & 44.93 & I \\
\hline 61.82 & 56.97 & N \\
\hline 73.85 & 69.00 & N \\
\hline 85.87 & 81.04 & N \\
\hline 97.90 & 93.08 & N \\
\hline 109.92 & 105.11 & N \\
\hline
\end{tabular}




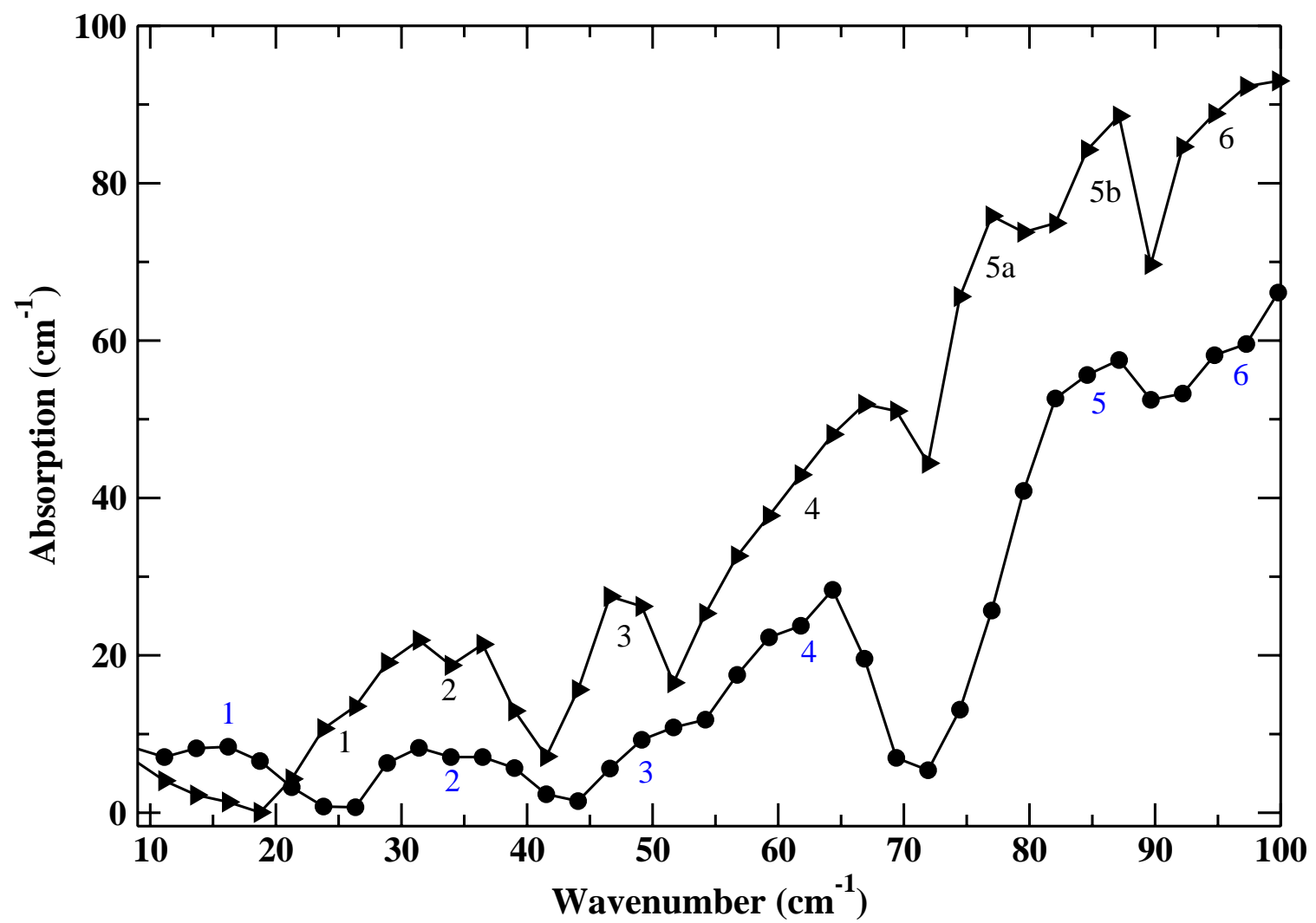

Figure 1 


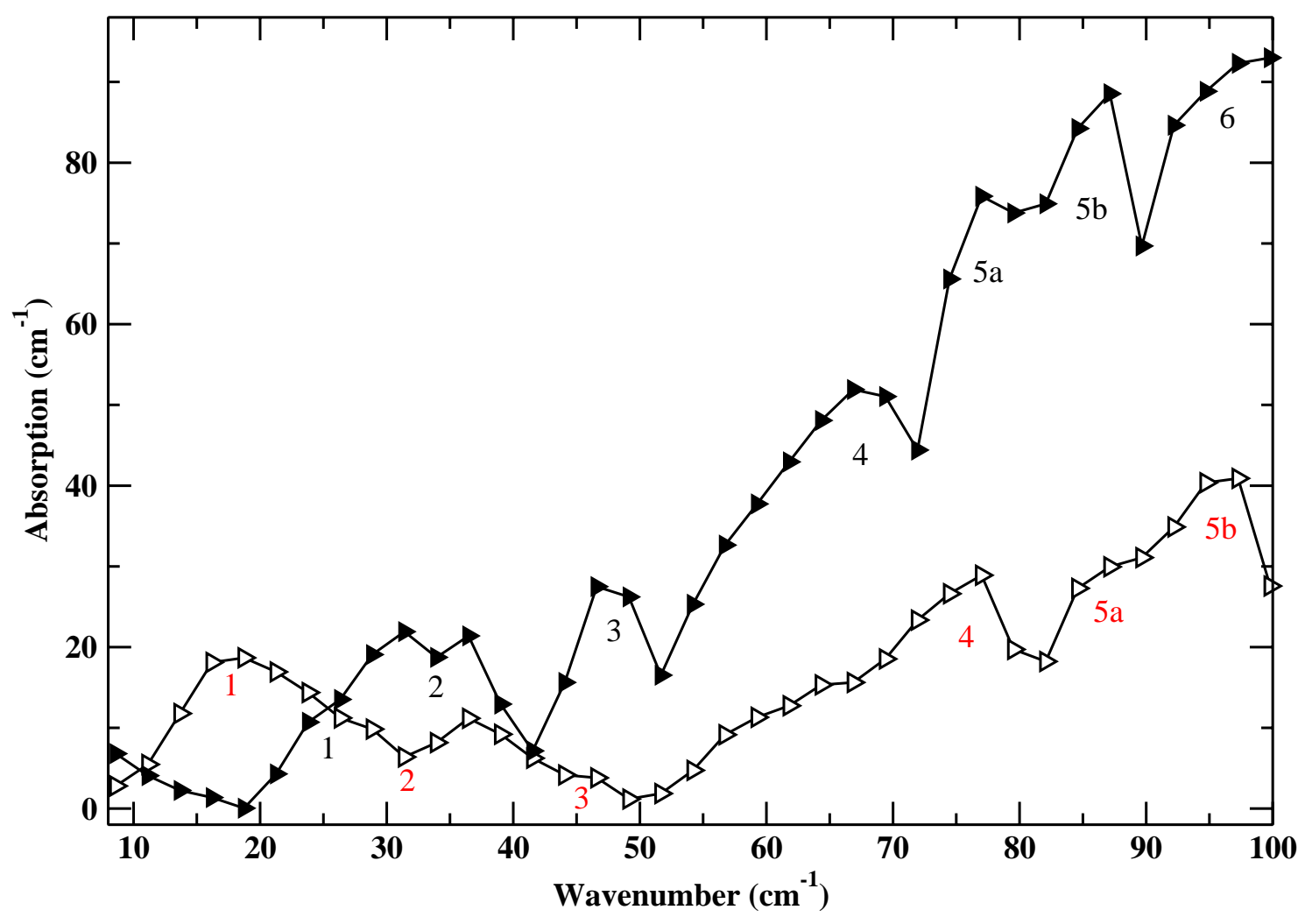

Figure 2 


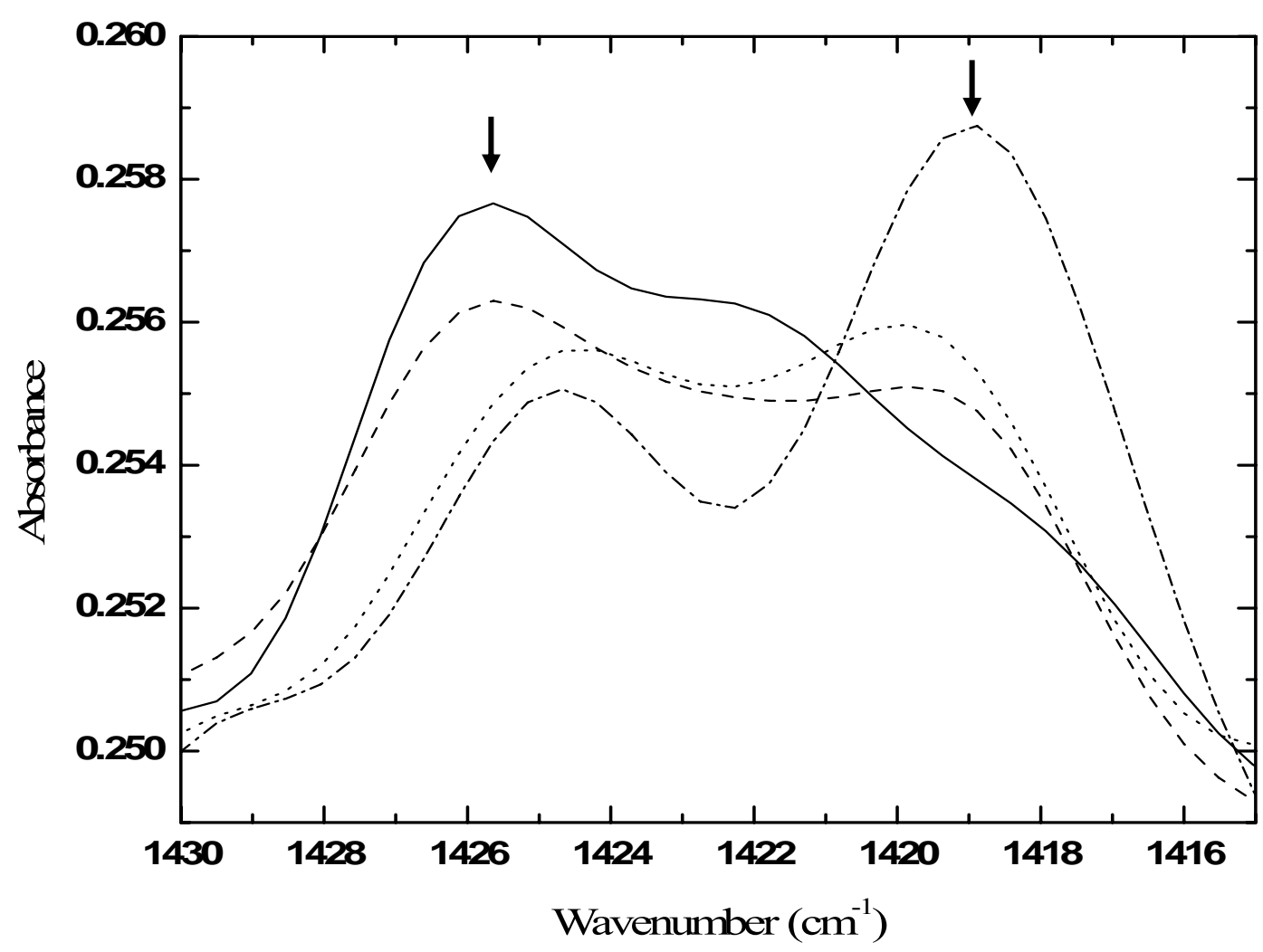

Figure 3 


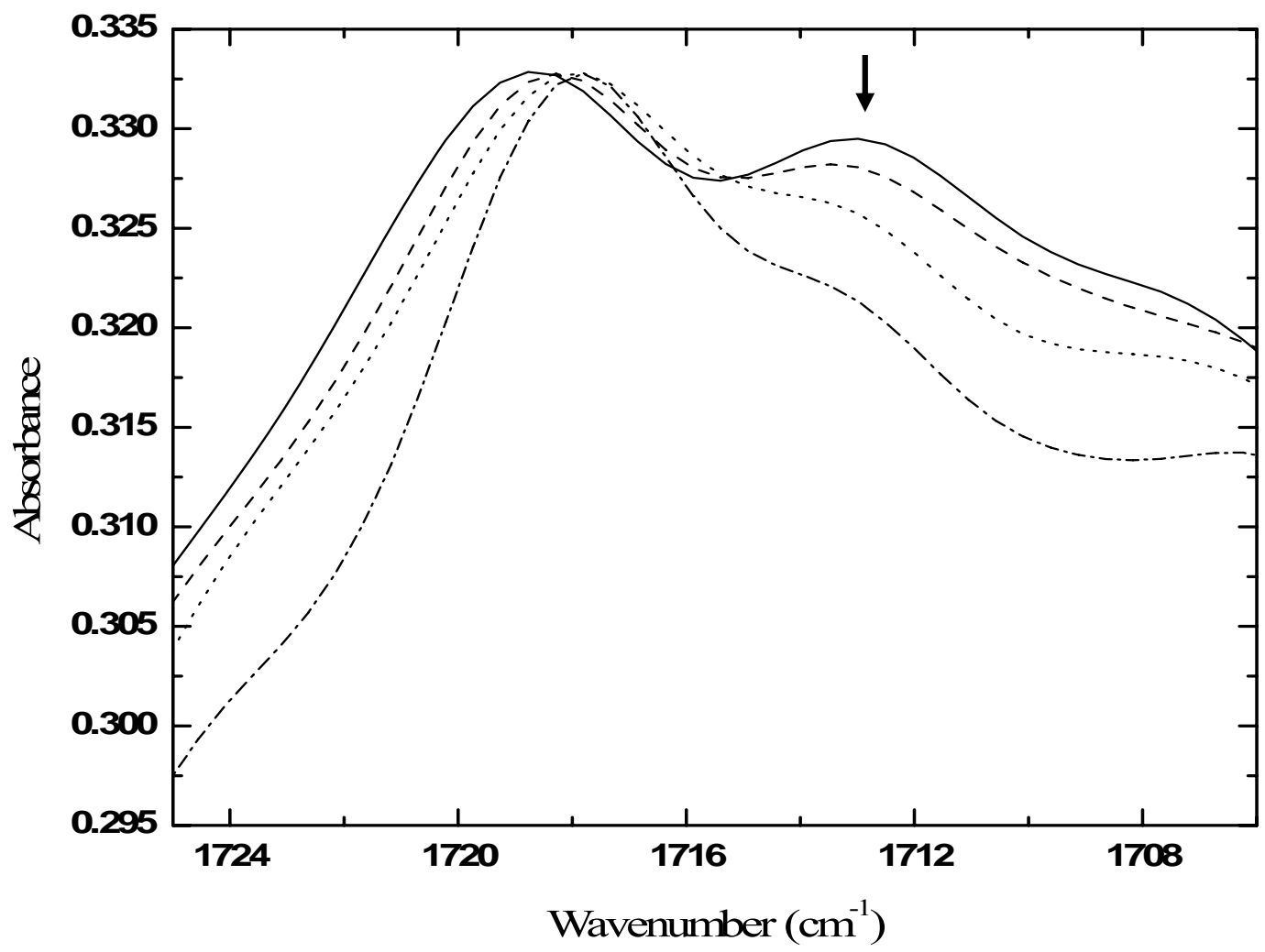

Figure 4 

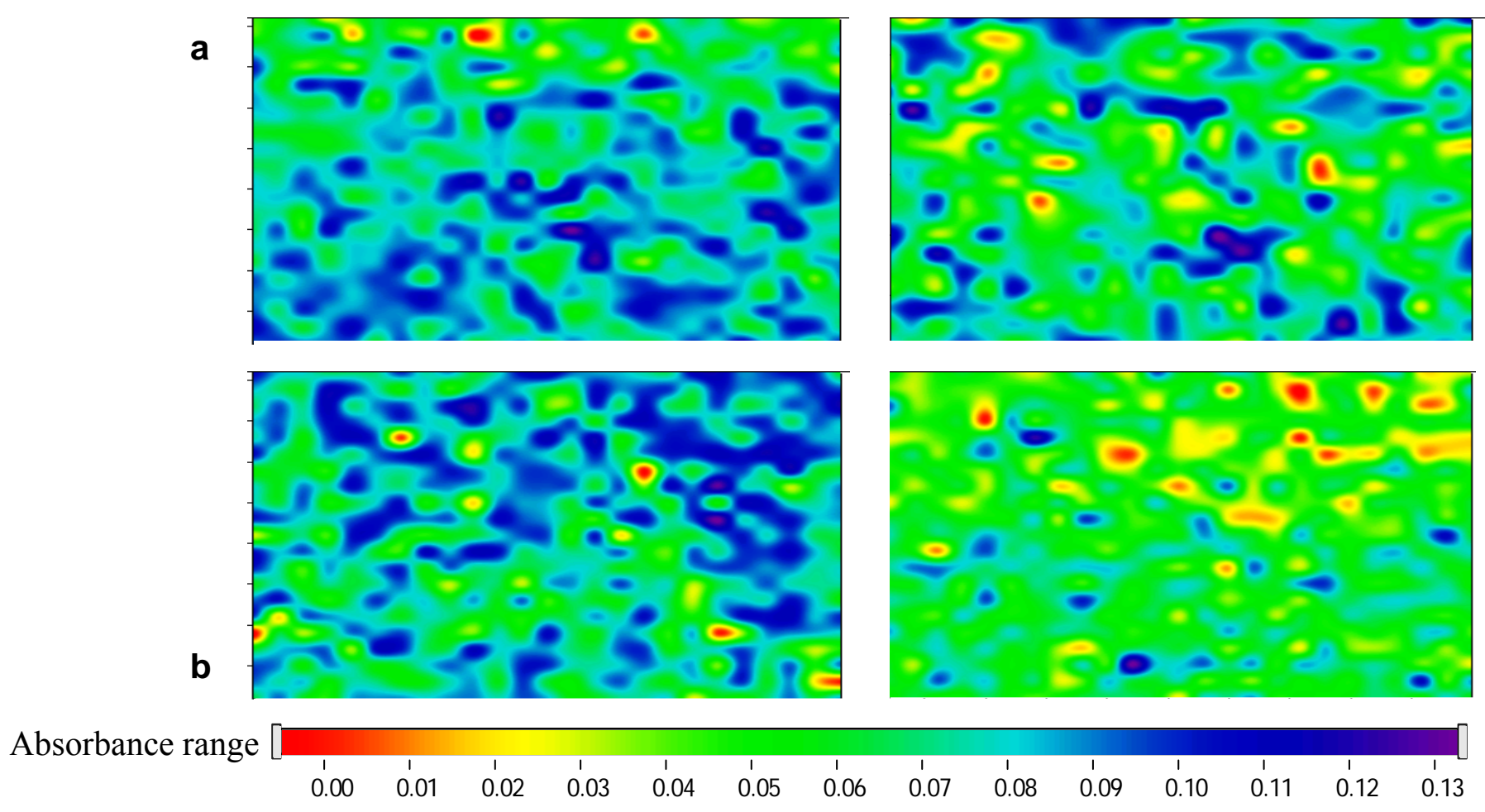

Figure 5 


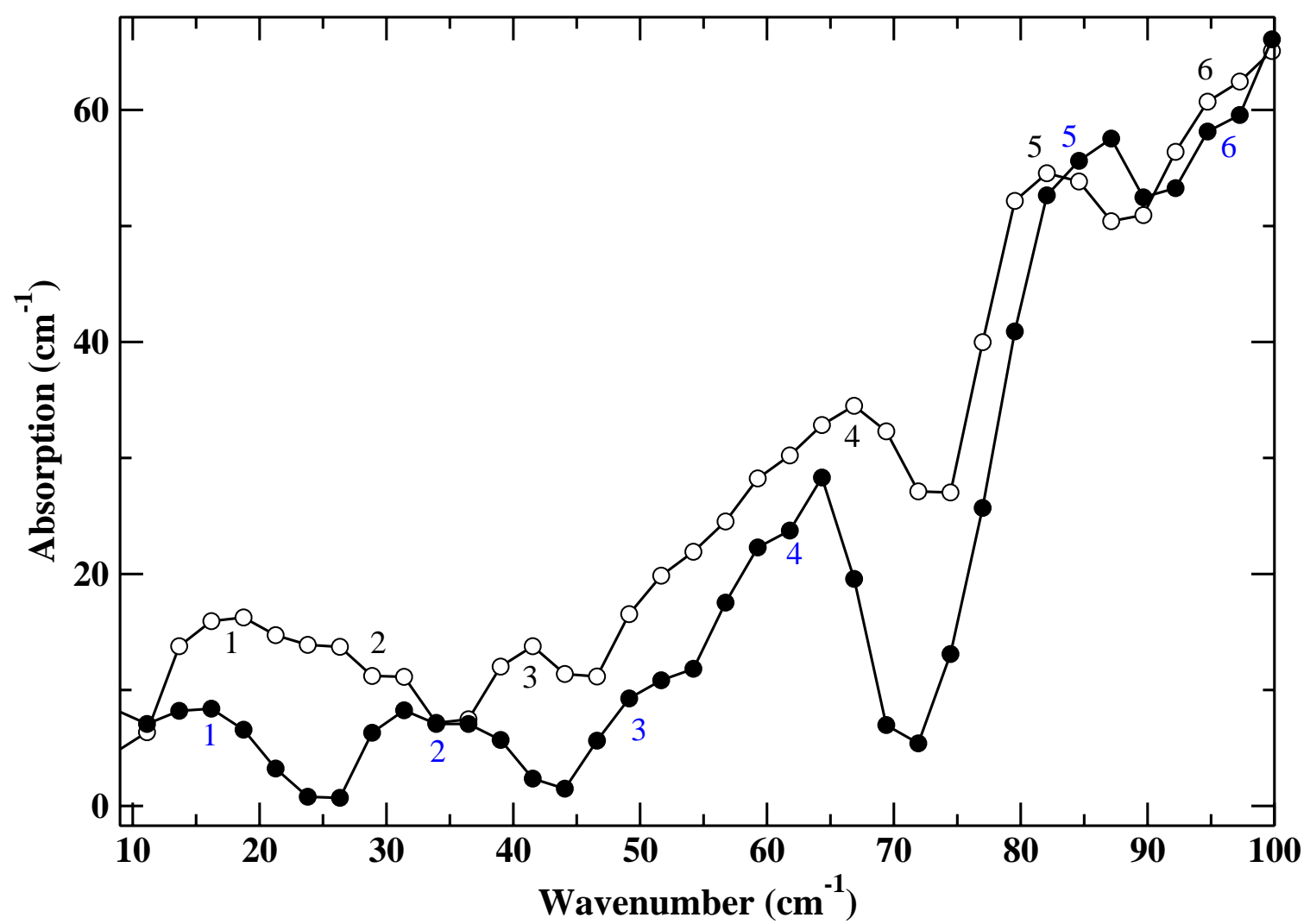

Figure 6 